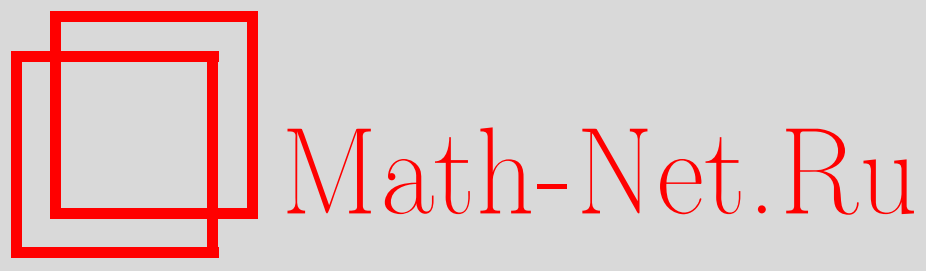

А. Г. Александров, Индекс векторных полей и логарифмические дифференциальные формы, Функи. анализ и его прил., 2005, том 39, выпуск 4, 1-13

DOI: https://doi.org/10.4213/faa81

Использование Общероссийского математического портала Math-Net.Ru подразумевает, что вы прочитали и согласны с пользовательским соглашением

http://www.mathnet.ru/rus/agreement

Параметры загрузки:

IP: 18.234 .197 .8

26 апреля 2023 г., 16:55:27

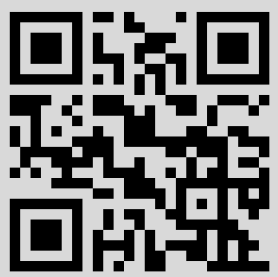


2005, т. 39, вып. 4, с. 1-13

УДК $515.16+512.7$

\title{
Индекс векторных полей и логарифмические дифференциальные формы*
}

\author{
(c) 2005. А. Г. АЛЕКСАНДРОВ
}

\section{Введение}

Индекс векторного поля - одно из самых первых понятий топологии и геометрии гладких многообразий, на свойствах индекса основываются важные результаты теории. Среди них - теорема Пуанкаре-Хопфа, согласно которой полный индекс векторного поля на замкнутом гладком ориентируемом многообразии не зависит от поля и равен характеристике Әйлера-Пуанкаре многообразия. При изучении многообразий с особенностями естественно поставить вопрос о существовании аналогичного инварианта в более общем контексте. Одно из возможных обобщений такого рода, первоначально возникшее в теории топологии слоений, оказалось хорошо приспособленным для использования в теории многообразий с особенностями. Соответствующий инвариант, названный в недавних работах $[5,6]$ гомологическим индексом, корректно определен для векторных полей, которые касаются многообразия в его неособых точках. Гомологический индекс обладает основными свойствами (см. [10]) классического топологического индекса и выражается через сумму локальных индексов Пуанкаре-Хопфа в особых точках векторных полей на гладкой деформации многообразия.

В настоящей статье излагается оригинальный метод вычисления гомологического индекса векторных полей на гиперповерхности, который опирается на теорию логарифмических дифференциальных форм и векторных полей. Главная идея нашего подхода состоит в том, что гомологический индекс можно описать в терминах мероморфных дифференциальных форм на объемлющем многообразии, которые обладают логарифмическими полюсами вдоль заданной гиперповерхности. Как мы увидим, систематическое использование теории логарифмических форм позволяет не только значительно упростить вычисления, но и прояснить смысл основных конструкций, на которых основаны многие работы, посвященные этой тематике.

После краткого обсуждения необходимых понятий и обозначений в статье вводится понятие логарифмического индекса векторного поля и доказывается, что гомологический индекс выражается через логарифмический. Затем оба инварианта описываются в терминах логарифмических дифференциальных форм в случае свободного дивизора Саито - гиперповерхности с неизолированными особенностями, а также вычисляются группы стягивающей гомологии комплекса регулярных голоморфных форм на такой гиперповерхности. В заключение

*Работа выполнена при частичной поддержке Российского фонда фундаментальных исследований (проект №02-01-00623). 
разобран случай нормальной гиперповерхности, в том числе и случай изолированной особенности. Кроме того, стягивающие гомологии комплекса регулярных мероморфных форм описываются с помощью вычета логарифмических форм.

\section{§1. Регулярные дифференциалы и векторные поля}

Пусть $M$ - комплексное многообразие размерности $n+1, n \geqslant 1$, a $\Omega_{M}^{q}, q \geqslant 0$, и $\operatorname{Der}(M)$ - пучки ростков голоморфных $q$-форм и векторных полей на $M$ соответственно. Пусть $D \subset M$ - дивизор, все неприводимые компоненты которого имеют кратность единица, так что в окрестности любой точки $x \in D$ локальное уравнение дивизора задается ростком голоморфной функции $h \in \mathscr{O}_{M, x}$ без кратных множителей: если $z=\left(z_{0}, \ldots, z_{n}\right)$ - система локальных координат в окрестности этой точки, то $h(z)=0$ - локальное уравнение дивизора $D$ в точке $x$ и $\mathscr{O}_{D, x} \cong \mathscr{O}_{M, x} /(h)$. Обозначим $\mathscr{O}_{D}$-модуль ростков регулярных (кэлеровых) дифференциалов порядка $q$ на $D$ через $\Omega_{D}^{q}$, так что

$$
\Omega_{D, x}^{q}=\Omega_{M, x}^{q} /\left(h \cdot \Omega_{M, x}^{q}+d h \wedge \Omega_{M, x}^{q-1}\right), \quad q \geqslant 0 .
$$

По аналогии с гладким случаем элементы из $\Omega_{D, x}^{q}$ обычно называют также ростками регулярных голоморфных форм на $D$ (ср. [11]). Пусть теперь $\operatorname{Der}(D)=$ $\operatorname{Hom}_{\mathscr{O}_{D}}\left(\Omega_{D}^{1}, \mathscr{O}_{D}\right)$ - пучок ростков регулярных векторных полей на $D$, и рассмотрим $V \in \operatorname{Der}(D)$. Обозначим через $\mathscr{V} \in \operatorname{Der}(M)$ голоморфное векторное поле на $M$, такое, что $\left.\mathscr{V}\right|_{D}=V$. Тогда внутреннее умножение (стягивание) векторных полей и дифференциальных форм $\iota \mathscr{V}: \Omega_{M}^{q} \rightarrow \Omega_{M}^{q-1}$ определяет структуру комплекса на $\Omega_{M}^{\bullet}$, поскольку $\iota_{\mathscr{V}}^{2}=0$. Стягивание $\iota \mathscr{V}$ индуцирует гомоморфизм $\mathscr{O}_{D}$-модулей $\iota_{V}: \Omega_{D}^{q} \rightarrow \Omega_{D}^{q-1}$, а также структуру комплекса на $\Omega_{D}^{\bullet}$. Соответствующие пучки и группы $\iota_{V}$-гомологий обозначаются через $H_{*}\left(\Omega_{D}^{\bullet}, \iota_{V}\right)$ и $H_{*}\left(\Omega_{D, x}^{\bullet}, \iota_{V}\right)$.

\section{§ 2. Гомологический индекс}

Если векторное поле $\mathscr{V}$ имеет изолированную особенность в точке $x \in D$, то группы $\iota_{V}$-гомологий комплекса $\Omega_{D, x}^{\bullet}-$ конечномерные векторные пространства, так что определена эйлерова характеристика

$$
\chi\left(\Omega_{D, x}^{\bullet}, \iota_{V}\right)=\sum_{i=0}^{n+1}(-1)^{i} \operatorname{dim} H_{i}\left(\Omega_{D, x}^{\bullet}, \iota_{V}\right)
$$

комплекса регулярных дифференциалов, которая называется гомологическим индексом векторного поля $V$ в точке $x \in D$ и обозначается через $\operatorname{Ind}_{\mathrm{hom}, D, x}(V)$. В неособых точках гиперповерхности $D$ гомологический индекс совпадает с топологическим индексом, или с локальным индексом Пуанкаре-Хопфа (см. $[5,6])$.

\section{§3. Логарифмические формы}

С дивизором $D$ можно связать $[13,14]$ когерентные аналитические пучки $\Omega_{M}^{q}(\log D), q>0$, и $\operatorname{Der}_{M}(\log D)$ следующим образом. Модуль $\Omega_{M, x}^{q}(\log D)$ состоит из ростков мероморфных $q$-форм $\omega$ на $M$ с полюсами вдоль $D$, таких, что $h \omega$ и $h d \omega$ голоморфны в точке $x$, т. е. $h \cdot \Omega_{M, x}^{q}(\log D) \subseteq \Omega_{M, x}^{q}$ и $h \wedge d \Omega_{M, x}^{q}(\log D) \subseteq$ 
$\Omega_{M, x}^{q+1}$. Последнее условие, очевидно, эквивалентно соотношению $d h \wedge \Omega_{M, x}^{q}(\log D)$ $\subseteq \Omega_{M, x}^{q+1}$. Модуль $\operatorname{Der}_{M, x}(\log D)$ состоит из ростков голоморфных векторных полей $\mathscr{V}$ на $M$, таких, что $\mathscr{V}(h) \in(h) \mathscr{O}_{M, x}$. Это, в частности, означает, что такое поле касается дивизора $D$ в его неособых точках и, кроме того, определяет регулярное векторное поле $V \in \operatorname{Der}(D)$ на $D$, так что $\left.\mathscr{V}\right|_{D}=V$. Внутреннее умножение полей и форм индуцирует спаривание $\mathscr{O}_{M}$-модулей

$$
\operatorname{Der}_{M}(\log D) \times \Omega_{M}^{q}(\log D) \longrightarrow \Omega_{M}^{q-1}(\log D),
$$

которое при $q=1$ является совершенным, а пучки $\operatorname{Der}_{M}(\log D)$ и $\Omega_{M}^{1}(\log D)$ оказываются двойственными $\mathscr{O}_{M}$-модулями.

Когерентный пучок $\mathscr{O}_{M}$-модулей $\Omega_{M}^{q}(\log D)$ называется пучком логарифмических q-форм, или пучком мероморфных $q$-форм с логарифмическими полюсами вдоль дивизора $D$, а $\operatorname{Der}_{M}(\log D)$ - пучком логарифмических векторных полей. По определению $\Omega_{M}^{0}(\log D) \cong \mathscr{O}_{M}$ и $\Omega_{M, x}^{n+1}(\log D) \cong \mathscr{O}_{M, x}\left(d z_{0} \wedge \cdots \wedge d z_{n} / h\right)$; при этом имеется включение $\Omega_{M}^{\bullet} \subseteq \Omega_{M}^{\bullet}(\log D)$, а вне дивизора $D$ имеют место естественные изоморфизмы, $\Omega_{M, x}^{\bullet} \cong \Omega_{M, x}^{\bullet}(\log D)$ для всех $x \notin D$.

\section{§4. Логарифмический индекс}

Рассмотрим векторное поле $\mathscr{V} \in \operatorname{Der}_{M}(\log D)$. Внутреннее умножение $\iota \mathscr{V}$ определяет структуру комплекса на $\Omega_{M}^{\bullet}(\log D)$.

ЛЕмма 1. Если все особенности векторного поля $\mathscr{V}$ изолированные, то группы цу-гомологий комплекса $\Omega_{M}^{\bullet}(\log D)$ - конечномерные векторные пространства.

Доказательство. Будем считать, что $M=\mathbb{C}^{n+1}$ и точка $x_{0}=0 \in D \subset M$ - изолированная особенность поля $\mathscr{V}$, так что $\mathscr{V}\left(x_{0}\right)=0$. Тогда $\mathscr{V}(x) \neq 0$ для любой точки $x$ из достаточно малой проколотой окрестности точки $x_{0}$ и в подходящей окрестности точки $x$ можно найти такую систему координат $\left(t, z_{1}^{\prime}, \ldots, z_{n}^{\prime}\right)$, в которой $\mathscr{V}=\partial / \partial t$. Так как $\mathscr{V}(h) \subseteq(h) \mathscr{O}_{M, 0}$, то $D \cong T \times D_{0}$, где через $T$ обозначен малый диск по переменной $t$, а $D_{0}-$ гиперповерхность в $\mathbb{C}^{n}$ (см., например, [12]). Нетрудно показать, что

$$
\Omega_{\mathbb{C}^{n+1}, 0}^{q}(\log D) \cong\left(\Omega_{\mathbb{C}^{n}, 0}^{q}\left(\log D_{0}\right) \oplus \Omega_{\mathbb{C}^{n}, 0}^{q-1}\left(\log D_{0}\right) \wedge d t\right) \otimes_{\mathbb{C}} \mathscr{O}_{\mathbb{C}, 0} .
$$

Действительно, для ростков голоморфных форм имеется изоморфизм $\Omega_{D, 0}^{q} \cong$ $\left(\Omega_{D_{0}, 0}^{q} \oplus \Omega_{D_{0}, 0}^{q-1} \wedge d t\right) \otimes_{\mathbb{C}} \mathscr{O}_{\mathbb{C}, 0}$, который легко получить из рассмотрения канонических проекций аналитического множества $T \times D_{0}$ на первый и второй сомножители и определения модуля $\Omega_{D, 0}^{q}$. Искомый изоморфизм для ростков логарифмических форм можно получить таким же рассуждением с использованием точной последовательности

$$
0 \rightarrow \Omega_{\mathbb{C}^{n+1}, 0}^{q}+\frac{d h}{h} \wedge \Omega_{\mathbb{C}^{n+1}, 0}^{q-1} \rightarrow \Omega_{\mathbb{C}^{n+1}, 0}^{q}(\log D) \stackrel{\cdot h}{\longrightarrow} \Omega_{D, 0}^{q} \rightarrow \Omega_{D, 0}^{q} / \text { Tors } \Omega_{D, 0}^{q} \rightarrow 0,
$$

которая получается из точной последовательности, выражающей подпучки кручения Tors $\Omega_{D}^{q}$ в терминах логарифмических дифференциальных форм; см. [1] или [3].

Далее, на $q$-м элементе комплекса $\left(\Omega_{M, 0}^{\bullet}(\log D), \iota \mathscr{V}\right)$ имеем

$$
\operatorname{Ker}\left(\iota_{\partial / \partial t}\right) \cong \operatorname{Im}\left(\iota_{\partial / \partial t}\right) \cong\left(\Omega_{\mathbb{C}^{n}, 0}^{q}\left(\log D_{0}\right) \oplus(0)\right) \otimes_{\mathbb{C}} \mathscr{O}_{\mathbb{C}, 0},
$$


т. е. соответствующие группы гомологий равны 0 для всех $q$. Если $x_{0} \in M \backslash D$, то без труда приходим к такому же заключению. Отсюда следует, что группы $\iota \mathscr{V}$-гомологий комплекса $\Omega_{M}^{\bullet}(\log D)$ могут быть нетривиальными лишь в особых точках поля. Из когерентности пучков логарифмических форм и их когомологий следует утверждение леммы.

ЗАмЕчАниЕ 1. Здесь, по существу, воспроизводится рассуждение из работы [6], где доказывается конечномерность групп $\iota_{V}$-гомологий комплекса дифференциалов $\Omega_{D, 0}^{\bullet}$. Однако приведенное доказательство содержит и новый элемент - изоморфизм (1).

Таким образом, если векторное поле $\mathscr{V}$ имеет изолированные особенности, то для любой точки $x \in M$ определена эйлерова характеристика

$$
\chi\left(\Omega_{M, x}^{\bullet}(\log D), \iota \mathscr{V}\right)=\sum_{i=0}^{n+1}(-1)^{i} \operatorname{dim} H_{i}\left(\Omega_{M, x}^{\bullet}(\log D), \iota \mathscr{V}\right),
$$

комплекса логарифмических дифференциальных форм, которую мы будем называть логарифмическим индексом поля $\mathscr{V}$ в точке $x$ и обозначать через $\operatorname{Ind}_{\log D, x}(\mathscr{V})$. Из предыдущего ясно, что $\operatorname{Ind}_{\log D, x}(\mathscr{V})=0$, если $\mathscr{V}(x) \neq 0$.

\section{§5. Индекс векторного поля на гиперповерхности}

Для изучения $\iota \mathscr{V}$-гомологий комплекса $\Omega_{D}^{\bullet}$ можно использовать подход, основанный на представлении регулярных голоморфных дифференциальных форм на гиперповерхности $D$ с помощью мероморфных форм на объемлющем многообразии с логарифмическими полюсами вдоль $D$ (см. [3]). Напомним (см. [1,3]), что для $q=0,1, \ldots, n+1$ существуют точные последовательности $\mathscr{O}_{M, x}$-модулей

$$
0 \longrightarrow \Omega_{M, x}^{q-1} / h \cdot \Omega_{M, x}^{q-1}(\log D) \stackrel{\wedge d h}{\longrightarrow} \Omega_{M, x}^{q} / h \cdot \Omega_{M, x}^{q} \longrightarrow \Omega_{D, x}^{q} \longrightarrow 0,
$$

где через $\wedge d h$ обозначается гомоморфизм внешнего умножения. Отсюда получаем точную последовательность комплексов

$$
0 \rightarrow\left(\Omega_{M, x}^{\bullet} / h \Omega_{M, x}^{\bullet}(\log D), \iota \mathscr{V}\right)[-1] \stackrel{\wedge d h}{\longrightarrow}\left(\Omega_{M, x}^{\bullet} / h \Omega_{M, x}^{\bullet}, \iota_{\mathscr{V}}\right) \longrightarrow\left(\Omega_{D, x}^{\bullet}, \iota_{V}\right) \rightarrow 0 .
$$

Действительно, тот факт, что умножение на $\wedge d h$ индуцирует морфизм комплексов, следует из тождества $\iota \mathscr{V}(\omega) \wedge d h=\iota \mathscr{V}(\omega \wedge d h)+(-1)^{q-1} \omega \wedge \mathscr{V}(h)$, поскольку второе слагаемое из правой части исчезает в факторкомплексе $\Omega_{M}^{\bullet} / h \Omega_{M}^{\bullet}$ ввиду условия $\mathscr{V}(h) \in(h) \mathscr{O}_{M, x}$. Теперь заметим, что из точной последовательности комплексов

$$
0 \longrightarrow\left(\Omega_{M, x}^{\bullet}, \iota \mathscr{V}\right) \stackrel{\cdot h}{\longrightarrow}\left(\Omega_{M, x}^{\bullet}, \iota \mathscr{V}\right) \longrightarrow\left(\Omega_{M, x}^{\bullet} / h \Omega_{M, x}^{\bullet}, \iota \mathscr{V}\right) \longrightarrow 0
$$

следует, что $\chi\left(\Omega_{M, x}^{\bullet} / h \Omega_{M, x}^{\bullet}, \iota \mathscr{V}\right)=0$. Таким образом, из точной последовательности (2) получаем

$$
\operatorname{Ind}_{\mathrm{hom}, D, x}(V)=-\chi\left(\left(\Omega_{M, x}^{\bullet} / h \Omega_{M, x}^{\bullet}(\log D), \iota \mathscr{V}\right)[-1]\right)=\chi\left(\Omega_{M, x}^{\bullet} / h \Omega_{M, x}^{\bullet}(\log D), \iota \mathscr{V}\right) .
$$

ПРЕДЛОЖЕНИЕ 1. Пусть $x \in D-$ изолированная особенность векторного поля $\mathscr{V} \in \operatorname{Der}(\log D)$, ростки $\mathscr{V}_{i} \in \mathscr{O}_{M, x}$ задаются разложением $\mathscr{V}=\sum_{i} \mathscr{V}_{i} \partial / \partial z_{i}$ u $J_{x} \mathscr{V}=\left(\mathscr{V}_{0}, \ldots, \mathscr{V}_{n}\right) \mathscr{O}_{M, x}$. Тогда

$$
\operatorname{Ind}_{\text {hom }, D, x}(V)=\operatorname{dim} \mathscr{O}_{M, x} / J_{x} \mathscr{V}-\operatorname{Ind}_{\log D, x}(V) .
$$


Доказательство. Рассмотрим точную последовательность

$$
0 \longrightarrow\left(\Omega_{M, x}^{\bullet}(\log D), \iota \mathscr{V}\right) \stackrel{\cdot h}{\longrightarrow}\left(\Omega_{M, x}^{\bullet}, \iota \mathscr{V}\right) \longrightarrow\left(\Omega_{M, x}^{\bullet} / h \Omega_{M, x}^{\bullet}(\log D), \iota \mathscr{V}\right) \longrightarrow 0 .
$$

Сначала заметим, что комплекс $\left(\Omega_{M, x}^{\bullet}, \iota \mathscr{V}\right)$ естественно изоморфен комплек-

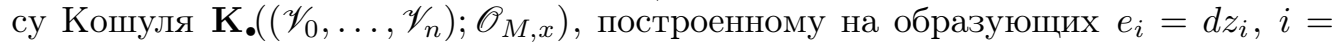
$0, \ldots, n$. Поскольку $x \in D-$ изолированная особенность векторного поля $\mathscr{V}$, последовательность $\left(\mathscr{V}_{0}, \ldots, \mathscr{V}_{n}\right)$ является $\mathscr{O}_{M, x}$-регулярной, так что комплекс Кошуля ацикличен во всех размерностях, кроме нулевой: $H_{0}\left(\mathbf{K}_{\bullet}\right) \cong \mathscr{O}_{M, x} / J_{x} \mathscr{V}$. Таким образом, соотношение

$$
\chi\left(\Omega_{M, x}^{\bullet} / h \Omega_{M, x}^{\bullet}(\log D), \iota \mathscr{V}\right)=\operatorname{dim}\left(\mathscr{O}_{M, x} / J_{x} \mathscr{V}\right)-\operatorname{Ind}_{\log D, x}(V)
$$

для эйлеровых характеристик комплексов из диаграммы (5) и тождество (4) дают утверждение предложения.

СлЕДСТВИЕ 1. В условиях предложения 1 имеются канонические изомор$\oint и з м ы$

$$
H_{i}\left(\Omega_{M, x}^{\bullet} / h \Omega_{M, x}^{\bullet}(\log D), \iota \mathscr{V}\right) \cong H_{i-1}\left(\Omega_{M, x}^{\bullet}(\log D), \iota \mathscr{V}\right), \quad i=2, \ldots, n+1 .
$$

Кроме того, тривиалъны следующие стариие группы гомологий:

$$
\begin{gathered}
H_{n+1}\left(\Omega_{M, x}^{\bullet} / h \Omega_{M, x}^{\bullet}(\log D), \iota \mathscr{V}\right), \quad H_{n}\left(\Omega_{M, x}^{\bullet} / h \Omega_{M, x}^{\bullet}(\log D), \iota \mathscr{V}\right), \\
H_{n+1}\left(\Omega_{M, x}^{\bullet}(\log D), \iota \mathscr{V}\right), \quad H_{n}\left(\Omega_{M, x}^{\bullet}(\log D), \iota \mathscr{V}\right), \quad H_{n-1}\left(\Omega_{M, x}^{\bullet}(\log D), \iota \mathscr{V}\right),
\end{gathered}
$$

а младиие группь єу-гомологий $H_{0}$ u $H_{1}$ комплекса $\Omega_{M, x}^{\bullet} / h \Omega_{M, x}^{\bullet}(\log D)$ включаются в точную последовательность

$$
0 \longrightarrow H_{1} \longrightarrow H_{0}\left(\Omega_{M, x}^{\bullet}(\log D), \iota \mathscr{V}\right) \stackrel{\cdot h}{\longrightarrow} \mathscr{O}_{M, x} / J_{x} \mathscr{V} \longrightarrow H_{0} \longrightarrow 0
$$

ДокАзАтЕЛЬство. Существование выписанных изоморфизмов и точной последовательности следует из ацикличности комплекса $\left(\Omega_{M, x}^{\bullet}, \iota \mathscr{V}\right)$ в положительных размерностях и точной последовательности (5). Равенство нулю первой группы списка следует из того, что модуль $\Omega_{M, x}^{n+1} / h \Omega_{M, x}^{n+1}(\log D)$ тривиален. Далее, из длинной точной последовательности гомологий, ассоциированной с последовательностью $(3)$, и из ацикличности комплекса $\left(\Omega_{M, x}^{\bullet}, \iota \mathscr{V}\right)$ в положительных размерностях следует, что комплекс $\left(\Omega_{M, x}^{\bullet} / h \Omega_{M, x}^{\bullet}, \iota \mathscr{V}\right)$ ацикличен во всех размерностях, кроме 0 и 1 . Поэтому равенство нулю второй группы получается из точной последовательности (2), а четвертой и пятой - из только что доказанных изоморфизмов для $i=n+1$ и $i=n$ соответственно. Наконец, тривиальность третьей группы списка следует из ацикличности комплекса $\left(\Omega_{M, x}^{\bullet}, \iota \mathscr{V}\right)$ в положительных размерностях и из точной последовательности (5).

Теперь можно сделать и первый шаг для вычисления $\iota_{V}$-гомологии комплекса $\Omega_{D, x}^{\bullet}$ регулярных дифференциалов.

УТВЕРЖДЕНИЕ 1. В условиях предложения 1 имеются канонические изоморбизмы

$$
\begin{gathered}
H_{0}\left(\Omega_{D, x}^{\bullet}, \iota_{V}\right) \cong \mathscr{O}_{M, x} /\left(h, J_{x} \mathscr{V}\right), \quad H_{3}\left(\Omega_{D, x}^{\bullet}, \iota_{V}\right) \cong H_{1}\left(\Omega_{M, x}^{\bullet} / h \Omega_{M, x}^{\bullet}(\log D), \iota_{\mathscr{V}}\right), \\
H_{i}\left(\Omega_{D, x}^{\bullet}, \iota_{V}\right) \cong H_{i-3}\left(\Omega_{M, x}^{\bullet}(\log D), \iota_{\mathscr{V}}\right), \quad i=4, \ldots, n+1,
\end{gathered}
$$


a группь гомологий $H_{1}$ и $H_{2}$ описываются с помощъю следующей четырехчленной точной последовательности:

$$
\begin{aligned}
0 & \longrightarrow H_{2}\left(\Omega_{D, x}^{\bullet}, \iota_{V}\right) \longrightarrow H_{0}\left(\Omega_{M, x}^{\bullet} / h \Omega_{M, x}^{\bullet}(\log D), \iota_{\mathscr{V}}\right) \\
& \stackrel{\wedge d h}{\longrightarrow} H_{1}\left(\Omega_{M, x}^{\bullet} / h \Omega_{M, x}^{\bullet}, \iota \mathscr{V}\right) \longrightarrow H_{1}\left(\Omega_{D, x}^{\bullet}, \iota_{V}\right) \longrightarrow 0
\end{aligned}
$$

ДокАЗАтЕЛЬство. Из начального фрагмента точной последовательности гомологий, ассоциированной с последовательностью (2), получаем первый изоморфизм, поскольку $H_{0}\left(\Omega_{M, x}^{\bullet} / h \Omega_{M, x}^{\bullet}(\log D)[-1]\right)=H_{-1}\left(\Omega_{M, x}^{\bullet} / h \Omega_{M, x}^{\bullet}(\log D)\right)=0$.

Далее, как отмечалось выше, комплекс $\left(\Omega_{M, x}^{\bullet} / h \Omega_{M, x}^{\bullet}, \iota \mathscr{V}\right)$ ацикличен во всех размерностях, кроме 0 и 1 . Две его нетривиальные группы гомологий находятся из точной последовательности

$$
0 \longrightarrow H_{1} \longrightarrow \mathscr{O}_{M, x} / J_{x} \mathscr{V} \stackrel{\cdot h}{\longrightarrow} \mathscr{O}_{M, x} / J_{x} \mathscr{V} \longrightarrow H_{0} \longrightarrow 0 .
$$

В частности, размерности этих групп гомологий как векторных пространств равны и имеются изоморфизмы $H_{0} \cong \mathscr{O}_{M, x} /\left(h, J_{x} \mathscr{V}\right)$ и $H_{1} \cong \operatorname{Ann}_{\mathscr{O}_{M, x} / J_{x} \mathscr{V}}(h)$.

Наконец, из точной последовательности гомологий, ассоциированной с последовательностью (2), и из условия ацикличности получаем изоморфизмы

$$
H_{i}\left(\Omega_{D, x}^{\bullet}, \iota_{V}\right) \cong H_{i-2}\left(\Omega_{M, x}^{\bullet} / h \Omega_{M, x}^{\bullet}(\log D), \iota \mathscr{V}\right), \quad i=3, \ldots, n+1,
$$

и точную последовательность (7). Эти изоморфизмы с учетом следствия 1 дают требуемое.

Таким образом, задача вычисления гомологического индекса и, в самом общем контексте, групп $\iota_{V}$-гомологий комплекса $\Omega_{D, x}^{\bullet}$ сводится к вычислению логарифмического индекса и групп $\iota \mathscr{v}$-гомологий комплекса логарифмических дифференциальных форм $\Omega_{M, x}^{\bullet}(\log D)$.

\section{§6. Свободные дивизоры}

Нетрудно описать гомологии комплекса логарифмических дифференциальных форм $\left(\Omega_{M, x}^{\bullet}(\log D), \iota \mathscr{v}\right)$ в случае, когда $D-$ свободный дивизор Саито, т. е. когда $\Omega_{M}^{q}(\log D)$ - локально свободные $\mathscr{O}_{M}$-модули для всех $q \geqslant 0$. Перед тем как приступить к вычислениям, напомним несколько известных фактов из теории свободных дивизоров. Так, в этом случае, согласно критерию Саито [14], для любой точки $x \in D$ существует набор ростков векторных полей $V^{0}, \ldots, V^{n} \in \operatorname{Der}_{M, x}(\log D)$, таких, что детерминант матрицы $\left\|v_{i j}\right\|$, образованной коэффициентами этих полей, равен $\gamma h$, где $\gamma$ - обратимый элемент из $\mathscr{O}_{M, x}$. Рассмотрим дифференциальные 1-формы

$$
\vartheta_{i}=\sum_{j=0}^{n} \Upsilon_{i j} d z_{j}, \quad i=0, \ldots, n,
$$

где $\Upsilon_{i j}$ - алгебраические дополнения элементов $v_{i j}$. Пусть $\Delta=\operatorname{det}\left\|v_{i j}\right\|$. Тогда дифференциальные формы $\omega_{i}=\vartheta_{i} / \Delta, i=0, \ldots, n$, образуют базис свободного $\mathscr{O}_{M, x}$-модуля $\Omega_{M, x}^{1}(\log D)$. Нетрудно проверить, что базисы $V^{0}, \ldots, V^{n}$ и $\omega_{0}, \ldots, \omega_{n}$ двойственны относительно совершенного спаривания из $\S 3$. В частности, $\iota_{V^{j}}\left(\omega_{i}\right)=\delta_{i}^{j}$ для $i, j=0, \ldots, n$, где $\delta_{i}^{j}-$ символ Кронекера, и $d f=$ 
$\sum_{i=0}^{n} \frac{\partial f}{\partial z_{i}} d z_{i}=\sum_{i=0}^{n} V^{i}(f) \omega_{i}$, если $f \in \mathscr{O}_{M, x}$. В дальнейшем будем предполагать, что $\Delta=h$. Это упростит обозначения и, как нетрудно видеть, не повлияет на результаты вычислений.

Вернемся к точной последовательности (5). Как уже отмечалось, комплекс в середине естественно изоморфен комплексу Кошуля К.((秎, ., , $\left.\left.\mathscr{V}_{n}\right) ; \mathscr{O}_{M, x}\right)$, построенному на образующих $e_{i}=d z_{i}, i=0, \ldots, n$. Если $D-$ свободный дивизор, то комплекс $\left(\Omega_{M, x}^{\bullet}(\log D), \iota \mathscr{V}\right)$ в точности по тем же соображениям естественно изоморфен комплексу Кошуля К. $\left(\left(\alpha_{0}, \ldots, \alpha_{n}\right) ; \mathscr{O}_{M, x}\right)$, построенному на образующих $e_{i}^{\prime}=\omega_{i}, i=0, \ldots, n$, где ростки $\alpha_{i} \in \mathscr{O}_{M, x}$ определяются из разложения векторного поля $\mathscr{V}$ по базисным векторным полям: $\mathscr{V}=\sum_{i} \alpha_{i} V^{i}$. Таким образом,

$$
H_{*}\left(\Omega_{M, x}^{\bullet}(\log D), \iota \mathscr{V}\right) \cong H_{*}\left(\mathbf{K}_{\bullet}\left(\left(\alpha_{0}, \ldots, \alpha_{n}\right) ; \mathscr{O}_{M, x}\right)\right)
$$

Нетрудно проверить, что умножение на $h$ в последовательности (5) индуцирует морфизм соответствующих комплексов Кошуля, который также происходит из умножения на $h$ в кольце коэффициентов. Действительно, обозначим через $\mathscr{A}_{0}$ гомоморфизм умножения на $h$ нульмерных компонент обоих комплексов, отождествленных с кольцом коэффициентов:

$$
\mathbf{K}_{0}\left(\left(\alpha_{0}, \ldots, \alpha_{n}\right) ; \mathscr{O}_{M}\right) \cong \mathscr{O}_{M} \cong \mathbf{K}_{0}\left(\left(\mathscr{V}_{0}, \ldots, \mathscr{V}_{n}\right) ; \mathscr{O}_{M}\right)
$$

Отображение $\mathscr{A}_{1}$ на одномерных компонентах задается по правилу $\mathscr{A}_{1}\left(e_{i}^{\prime}\right)=$ $\sum_{j} \Upsilon_{i j} e_{j}$. При этом $\iota \mathscr{V} \mathscr{A}_{1}\left(e_{i}^{\prime}\right)=\mathscr{A}_{0} \iota \mathscr{V}\left(e_{i}^{\prime}\right)=h \alpha_{i}$, поскольку

$$
\begin{aligned}
\iota_{\mathscr{V}} \mathscr{A}_{1}\left(e_{i}^{\prime}\right) & =\iota_{\mathscr{V}}\left(\sum_{j} \Upsilon_{i j} e_{j}\right)=\sum_{j} \Upsilon_{i j} \iota_{\mathscr{V}}\left(e_{j}\right)=\sum_{j} \Upsilon_{i j} \mathscr{V}_{j} \\
& =\sum_{j} \Upsilon_{i j} \sum_{k}\left(\alpha_{k} v_{k j}\right)=\sum_{k} \alpha_{k}\left(\sum_{j} \Upsilon_{i j} v_{k j}\right)=h \alpha_{i}=\mathscr{A}_{0} \iota_{\mathscr{V}}\left(e_{i}^{\prime}\right) .
\end{aligned}
$$

В этой цепочке равенств третье следует из того, что $\mathscr{V}_{j}=\sum_{i} v_{i j} \alpha_{i}$ (ввиду тождеств $\left.\mathscr{V}=\sum_{i} \mathscr{V}_{i} \partial / \partial z_{i}=\sum_{i} \alpha_{i} V^{i}=\sum_{i, j} \alpha_{i} v_{i j} \partial / \partial z_{j}\right)$, а предпоследнее - из того, что $\sum_{j} \Upsilon_{i j} v_{k j}=\delta_{i}^{k} h$ по основным свойствам систем дуальных базисов. Остальные отображения $\mathscr{A}_{\ell}, \ell \geqslant 2$, определяются как внешние степени отображения $\mathscr{A}_{1}$. Суммируя сказанное выше, получаем

СлЕДСТВИЕ 2. Пусть $D-$ свободный дивизор Саито, $x \in D-$ изолированная особенность векторного поля $\mathscr{V} \in \operatorname{Der}(\log D)$, a $\mathscr{V}=\sum_{i} \alpha_{i} V^{i}-$ разложение этого поля по базисным векторным полям. Тогда

$$
\operatorname{Ind}_{\log D, x}(\mathscr{V})=\chi\left(\mathbf{K}_{\bullet}\left(\left(\alpha_{0}, \ldots, \alpha_{n}\right) ; \mathscr{O}_{M, x}\right)\right)
$$

ЗАМЕЧАНИЕ 2 . Пусть $J_{\log D, x} \mathscr{V}=\left(\alpha_{0}, \ldots, \alpha_{n}\right) \mathscr{O}_{M, x}$, и предположим, что коэффициенты $\left(\alpha_{0}, \ldots, \alpha_{n}\right)$ разложения векторного поля $\mathscr{V}$ по базисным полям образуют регулярную $\mathscr{O}_{M, x}$-последовательность. Тогда

$$
\operatorname{Ind}_{\text {hom }, D, x}(\mathscr{V})=\operatorname{dim} \mathscr{O}_{M, x} / J_{x} \mathscr{V}-\operatorname{dim} \mathscr{O}_{M, x} / J_{\log D, x} \mathscr{V}
$$




\section{§7. Группы $\iota_{V}$-гомологий комплекса регулярных дифференциалов}

Покажем, как группы гомологий комплексов $\Omega_{M, x}^{\bullet} / h \Omega_{M, x}^{\bullet}(\log D)$ и $\Omega_{D, x}^{\bullet}$ описываются в терминах комплекса Кошуля для свободных дивизоров. Начнем с анализа первого комплекса.

УтвеРЖДЕНИЕ 2. В условиях и обозначениях следствия 2 существуют канонические изоморфизмы

$$
\begin{gathered}
H_{0}\left(\Omega_{M, x}^{\bullet} / h \Omega_{M, x}^{\bullet}(\log D), \iota \mathscr{V}\right) \cong \mathscr{O}_{M, x} /\left(h, J_{x} \mathscr{V}\right), \\
H_{1}\left(\Omega_{M, x}^{\bullet} / h \Omega_{M, x}^{\bullet}(\log D), \iota \mathscr{V}\right) \cong \operatorname{Ann}_{\mathscr{O}_{M, x} / J_{x} \mathscr{V}}(h) / J_{\log D, x} \mathscr{V} \\
H_{i}\left(\Omega_{M, x}^{\bullet} / h \Omega_{M, x}^{\bullet}(\log D), \iota \mathscr{V}\right) \cong H_{i-1}\left(\mathbf{K}_{\bullet}\left(\left(\alpha_{0}, \ldots, \alpha_{n}\right) ; \mathscr{O}_{M, x}\right)\right), \quad i=2, \ldots, n+1 .
\end{gathered}
$$

ДокАЗАТЕЛЬСтво. Поскольку $H_{0}\left(\mathbf{K}_{.}\left(\left(\alpha_{0}, \ldots, \alpha_{n}\right) ; \mathscr{O}_{M, x}\right)\right) \cong \mathscr{O}_{M, x} / J_{\log D, x} \mathscr{V}$, точная последовательность (6) принимает вид

$$
0 \longrightarrow H_{1} \longrightarrow \mathscr{O}_{M, x} / J_{\log D, x} \mathscr{V} \stackrel{\cdot h}{\longrightarrow} \mathscr{O}_{M, x} / J_{x} \mathscr{V} \longrightarrow H_{0} \longrightarrow 0 .
$$

Из нее сразу получаем первые два изоморфизма, а для группы $H_{1}$ еще и такой: $H_{1} \cong(h) \cap J_{x} \mathscr{V} /(h) \cdot J_{\log D, x} \mathscr{V}$. Изоморфизмы для $i \geqslant 2$ получаются непосредственно из следствия 1.

ЗАмечАниЕ 3. Отметим, что морфизм умножения на $h$ отображает фактормодули в необычном направлении - из меньшего в больший. Действительно, как уже отмечалось, $\mathscr{V}_{j}=\sum_{i} v_{i j} \alpha_{i}$, так что $J_{x} \mathscr{V} \subseteq J_{\log D, x} \mathscr{V}$. Наконец, тот факт, что умножение на $h$ корректно определяет отображение фактормодулей, следует из включения $h J_{\log D, x} \mathscr{V} \subseteq J_{x} \mathscr{V}$, которое нетрудно проверить. А именно, в обозначениях предыдущего параграфа $\sum_{k} v_{j k} \Upsilon_{i k}=h \delta_{i}^{j}$ по основному свойству систем дуальных базисов. Отсюда следует, что $h \alpha_{i}=\sum_{j} h \delta_{i}^{j} \alpha_{j}=$ $\sum_{k} \sum_{j} \Upsilon_{i k} v_{j k} \alpha_{j}=\sum_{k} \Upsilon_{i k} \mathscr{V}_{k}$

Перейдем теперь к описанию гомологий комплекса регулярных дифференциалов на $D$.

Tеорема 1. Пусть $D-$ свободный дивизор Саито, $x \in D-$ изолированная особенность векторного поля $\mathscr{V} \in \operatorname{Der}(\log D)$ и $\mathscr{V}(h)=\varphi h, \varphi \in \mathscr{O}_{M, x}$. Обозначим через $\mathscr{B}_{x}$ локальное кольцо $\mathscr{O}_{M, x} / J_{x} \mathscr{V}$. Тогда имеются канонические изоморфизми

$$
\begin{gathered}
H_{0}\left(\Omega_{D, x}^{\bullet}, \iota_{V}\right) \cong \mathscr{O}_{M, x} /\left(h, J_{x} \mathscr{V}\right), \\
H_{1}\left(\Omega_{D, x}^{\bullet}, \iota_{V}\right) \cong \operatorname{Ann}_{\mathscr{B}_{x}}(h) /(\varphi) \mathscr{B}_{x}, \quad H_{2}\left(\Omega_{D, x}^{\bullet}, \iota_{V}\right) \cong \operatorname{Ann}_{\mathscr{B}_{x}}(\varphi) /(h) \mathscr{B}_{x}, \\
H_{i}\left(\Omega_{D, x}^{\bullet}, \iota_{V}\right) \cong H_{i-1}\left(\mathbf{K} \cdot\left(\left(\alpha_{0}, \ldots, \alpha_{n}\right) ; \mathscr{O}_{M, x}\right)\right), \quad i=3, \ldots, n+1 .
\end{gathered}
$$

ДоКАЗАТЕЛЬСТво. Из точной последовательности (2) следует, что

$$
H_{0}\left(\Omega_{D, x}^{\bullet}, \iota_{V}\right) \cong H_{0}\left(\Omega_{M, x}^{\bullet} / h \Omega_{M, x}^{\bullet}, \iota_{V}\right) \cong \mathscr{O}_{M, x} /\left(h, J_{x} \mathscr{V}\right) .
$$

Теперь докажем, что точную последовательность (7) можно представить в следующем виде:

$$
0 \rightarrow H_{2}\left(\Omega_{D, x}^{\bullet}, \iota_{V}\right) \longrightarrow \mathscr{O}_{M, x} /\left(h, J_{x} \mathscr{V}\right) \stackrel{\varphi}{\longrightarrow} \operatorname{Ann}_{\mathscr{B}_{x}}(h) \longrightarrow H_{1}\left(\Omega_{D, x}^{\bullet}, \iota_{V}\right) \rightarrow 0,
$$

где отображение в середине есть умножение на $\varphi \in \mathscr{O}_{M, x}$. Для этого рассмотрим кусок точной последовательности (7), состоящий из двух средних компонент. По 
предыдущему утверждению $H_{0}\left(\Omega_{M, x}^{\bullet} / h \Omega_{M, x}^{\bullet}(\log D), \iota \mathscr{V}\right) \cong \mathscr{O}_{M, x} /\left(h, J_{x} \mathscr{V}\right)$, так что этот фрагмент принимает вид

$$
\wedge d h: \mathscr{O}_{M, x} /\left(h, J_{x} \mathscr{V}\right) \rightarrow H_{1}\left(\Omega_{M, x}^{\bullet} / h \Omega_{M, x}^{\bullet}, \iota \mathscr{V}\right) \cong \operatorname{Ann}_{\mathscr{B}_{x}}(h) .
$$

Теперь опишем связывающий гомоморфизм $\wedge d h$ в явном виде. Для этого рассмотрим росток $g \in \mathscr{O}_{M, x} /\left(h, J_{x} \mathscr{V}\right)$, представляющий произвольный элемент 0 -мерной $\iota \mathscr{V}$-гомологии комплекса $\Omega_{M, x}^{\bullet} / h \Omega_{M, x}^{\bullet}(\log D)$. Тогда по определению

$$
\wedge d h(g)=g d h \in \operatorname{Ker}\left(\iota \mathscr{V}: \Omega_{M, x}^{1} / h \Omega_{M, x}^{1} \rightarrow \mathscr{O}_{M, x} / h \mathscr{O}_{M, x}\right) .
$$

Если также через $g$ обозначить росток в $\mathscr{O}_{M, x}-$ соответствующий представитель выбранного выше элемента 0-мерной группы гомологий, - то снова из определений выводим соотношение $\iota \mathscr{V}(g d h)=h G$ для некоторого $G \in \mathscr{O}_{M, x}$. Наконец, из определения связывающего гомоморфизма следует, что $\wedge d h$ сопоставляет элементу $g$ росток

$$
G=\iota_{\mathscr{V}}(g d h) / h=g \mathscr{V}(h) / h=g \varphi \in \operatorname{Ann}_{\mathscr{B}_{x}}(h) \cong H_{1}\left(\Omega_{M, x}^{\bullet} / h \Omega_{M, x}^{\bullet}, \iota \mathscr{V}\right),
$$

что и требовалось доказать.

В качестве еще одного непосредственного следствия из точной последовательности $\left(7^{\circ}\right)$ получаем, что

$$
H_{2}\left(\Omega_{D, x}^{\bullet}, \iota_{V}\right) \cong\left(J_{x} \mathscr{V}: \varphi\right) /\left(h, J_{x} \mathscr{V}\right), \quad H_{1}\left(\Omega_{D, x}^{\bullet}, \iota_{V}\right) \cong \operatorname{Ann}_{\mathscr{B}_{x}}(h) /(\varphi) \mathscr{B}_{x},
$$

где через $\left(J_{x} \mathscr{V}: \varphi\right)$ обозначается дробный идеал, состоящий из ростков $f \in \mathscr{O}_{M, x}$, таких, что $f \varphi \in J_{x} \mathscr{V}$. В частности, $\left(J_{x} \mathscr{V}: \varphi\right) /\left(h, J_{x} \mathscr{V}\right) \cong \operatorname{Ann}_{\mathscr{B}_{x}}(\varphi) /(h) \mathscr{B}_{x}$. Полезно еще отметить, что если $\varphi \equiv 0$, то четырехчленная последовательность $\left(7^{\circ}\right)$ распадается на два изоморфизма

$$
H_{2}\left(\Omega_{D, x}^{\bullet}, \iota_{V}\right) \cong \mathscr{O}_{M, x} /\left(h, J_{x} \mathscr{V}\right), \quad H_{1}\left(\Omega_{D, x}^{\bullet}, \iota_{V}\right) \cong \operatorname{Ann}_{\mathscr{B}_{x}}(h) .
$$

ПрЕДЛОЖЕНИЕ 2. Предположим, что коэфбициенты $\left(\alpha_{0}, \ldots, \alpha_{n}\right)$ разложения векторного поля $\mathscr{V}$ по базисным полям образуют регулярную последовательность. Тогда

$$
\operatorname{Ind}_{\text {hom }, D, x}(\mathscr{V})=\operatorname{dim} \mathscr{O}_{M, x} /\left(h, J_{x} \mathscr{V}\right)
$$

ДокАЗАтельство. По доказанной теореме группы $\iota_{V}$-гомологий $H_{i}$ комплекса $\Omega_{D, x}^{\bullet}$ равны нулю при $i \geqslant 3$, а пространства групп гомологий $H_{1}$ и $H_{2}$ этого комплекса имеют одинаковую размерность. Последнее прямо следует из сравнения размерностей всех членов точных последовательностей $\left(6^{\circ}\right)$ и $\left(7^{\circ}\right)$.

СлЕДСТВИЕ 3. Предположим, что оба идеала $J_{x} \mathscr{V} \subset J_{\log D, x} \mathscr{V}$ порождаются регулярными последовательностями, причем детерминант матрицы перехода равен $h$. Тогда группа гомологий $H_{1}$ в диаграмме (6) тривиальна, т.е. гомоморфизм умножсения на $h$ в этой точной последовательности инвективен.

ДокАЗАТЕЛЬСтво. Из двух выражений для гомологического индекса, полученных в предложении 2 и в замечании 2 (формула (8)) из предыдущего параграфа, следует, что

$$
\operatorname{dim} \mathscr{O}_{M, x} /\left(h, J_{x} \mathscr{V}\right)=\operatorname{dim} \mathscr{O}_{M, x} / J_{x} \mathscr{V}-\operatorname{dim} \mathscr{O}_{M, x} / J_{\log D, x} \mathscr{V}
$$

Изоморфизм $H_{0} \cong \mathscr{O}_{M, x} /\left(h, J_{x} \mathscr{V}\right)$ показывает, что $H_{1}=0$, а это и требовалось доказать. 
ЗАмечАниЕ 4. На самом деле тот факт, что при аналогичных условиях умножение на $h$ инъективно, - ключевой момент в доказательстве теоремы локальной двойственности Гротендика в инвариантной форме (см. [8]). Там же это утверждение проверяется другим способом, с помощью вычисления функторов Ext.

ПримеРЫ. Найдем теперь индекс и группы гомологий для двух примеров (5.1) и (5.3) из работы [6]. В первом примере $M=\left(\mathbb{C}^{3}, 0\right)$ и гиперповерхность $D \subset M$ задается функцией $h\left(z_{0}, z_{1}, z_{2}\right)=z_{1} z_{2}$. Заметим, что $D-$ свободный дивизор Саито. Действительно, пусть $\partial_{i}=\partial / \partial z_{i}$. Тогда $V^{0}=\partial_{0}$, $V^{1}=z_{1} \partial_{1}+z_{2} \partial_{2}, V^{2}=z_{1} \partial_{1}-z_{2} \partial_{2}$ составляют базис модуля $\operatorname{Der}_{M}(\log D)$. Pacсмотрим семейство векторных полей $\mathscr{V}_{t}=z_{0}^{p} \partial_{0}+t z_{1} \partial_{1}+z_{2} \partial_{2}, p \geqslant 1$. Тогда $\mathscr{V}_{t} \in$ $\operatorname{Der}_{M}(\log D)$ имеет изолированные особенности для любого $t \in \mathbb{C} \backslash\{0\}$. Далее, $\mathscr{V}_{t}=z_{0}^{p} V^{0}+\frac{1}{2}(t+1) V^{1}+\frac{1}{2}(t-1) V^{2}$, так что идеал $J_{\log D, 0}=\left(z_{0}^{p}, t+1, t-1\right)$ порождается регулярной последовательностью, которая содержит обратимый элемент при любом значении параметра $t$. Из формулы (8) следует, что $\operatorname{Ind}_{\mathrm{hom}, D, 0}$ $=\operatorname{dim} H_{0}\left(\Omega_{D, 0}^{\bullet}\right)=\operatorname{dim} \mathscr{O}_{M, 0} /\left(z_{0}^{p}, z_{1}, z_{2}\right)=p$. Для вычисления остальных групп гомологий воспользуемся теоремой 1 . Тогда $H_{3}\left(\Omega_{D, 0}^{\bullet}\right)=0$. Так как $\mathscr{V}_{t}(h)=$ $(t+1) h$, то $\varphi_{t}=t+1$. Если $t=-1$, то $\varphi_{-1} \equiv 0$, так что $\operatorname{dim} H_{2}\left(\Omega_{D, 0}^{\bullet}\right)=$ $\operatorname{dim} \mathscr{O}_{M, 0} /\left(z_{0}^{p}, z_{1}, z_{2}\right)=p$ и, аналогично, $\operatorname{dim} H_{1}\left(\Omega_{D, 0}^{\bullet}\right)=p$. Если же $t \neq-1$, то $\varphi_{t}$ обратим, так что $H_{1}\left(\Omega_{D, 0}^{\bullet}\right)=H_{2}\left(\Omega_{D, 0}^{\bullet}\right)=0$.

Второй пример - это дискриминант минимальной версальной деформации простой особенности $A_{3}$. Хорошо известно, что такие дискриминанты - свободные дивизоры [13]. В тех же обозначениях дискриминант $D \subset M$ задается функцией $h=256 z_{0}^{3}-128 z_{0}^{2} z_{2}^{2}+16 z_{0} z_{2}^{4}+144 z_{0} z_{1}^{2} z_{2}-4 z_{1}^{2} z_{2}^{3}-27 z_{1}^{4}$. Тогда $V^{0}=4 \partial_{0}+3 \partial_{1}+2 \partial_{2}, V^{1}=-\frac{1}{2} z_{1} z_{2} \partial_{0}+\left(4 z_{0}-z_{2}^{2}\right) \partial_{1}+3 z_{1} \partial_{2}$ и $V^{2}=-\frac{3}{4} z_{1}^{2} \partial_{0}-$ $2 z_{1} z_{2} \partial_{1}+\left(4 z_{0}-z_{2}^{2}\right) \partial_{2}$ образуют базис модуля $\operatorname{Der}_{M}(\log D)$. Рассмотрим семейство векторных полей $\mathscr{V}_{t}=z_{2} V^{0}+t V^{2}$. Тогда $\mathscr{V}_{t} \in \operatorname{Der}_{M}(\log D)$ имеет изолированные особенности для любого $t \in \mathbb{C} \backslash\left\{0, \frac{3}{2}, 2\right\}$. Здесь идеал $J_{\log D, 0}=\left(z_{2}, t\right)$ порождается регулярной последовательностью, которая содержит обратимый элемент при любом допустимом значении параметра $t$. Снова из формулы (8) следует, что $\operatorname{Ind}_{\text {hom }, D, 0}=\operatorname{dim} H_{0}\left(\Omega_{D, 0}^{\bullet}\right)=\operatorname{dim} \mathscr{O}_{M, 0} /\left(h, J_{0} \mathscr{V}_{t}\right)=\operatorname{dim} \mathscr{O}_{\mathbb{C}^{2}, 0} /\left(z_{1}^{2}-z_{2}^{3}, z_{1} z_{2}\right)=5$. По теореме 1 легко вычислить и две нетривиальные группы гомологий. Имеем $\mathscr{V}_{t}(h)=(12-4 t) z_{2}$, т. е. $\varphi_{t}=12-4 t$. Если $t=3$, то $\varphi_{3} \equiv 0$, так что $\operatorname{dim} H_{2}\left(\Omega_{D, 0}^{\bullet}\right)=\operatorname{dim} \mathscr{O}_{M, 0} /\left(h, J_{0} \mathscr{V}_{t}\right)=5$ и, аналогично, $\operatorname{dim} H_{1}\left(\Omega_{D, 0}^{\bullet}\right)=5$. Если же $t \neq 3$, то $\varphi_{t}$ обратим, так что $H_{1}\left(\Omega_{D, 0}^{\bullet}\right)=H_{2}\left(\Omega_{D, 0}^{\bullet}\right)=0$.

\section{§8. Нормальные гиперповерхности}

Обозначим через $Z=\operatorname{Sing} D$ подмножество особенностей приведенного дивизора $D$, а через $c=\operatorname{codim}(Z, D)$ коразмерность подмножества $Z$ в $D$. Хорошо известно (см. [2]), что для свободных дивизоров Саито $c=1$, т. е. в определенном смысле особенности дивизора $D$ составляют максимально возможное подмножество. Если же $c \geqslant 2$, то из критерия Серра следует, что гиперповерхность $D$ - нормальное многообразие (см. [9, гл. II, предложение 8.23]). Для дальнейшего анализа этого случая мы используем следующую переформулировку понятия логарифмической формы из работ $[13,14]$. 
Лемма 2. Росток $\omega$ мероморфной дифференииальной q-формь в точке $x \in M$ с полюсами вдоль $D$ является ростком логарифмической бормы, т. $е$. $\omega \in \Omega_{M, x}^{q}(\log D)$, тогда и только тогда, когда существуют ростки голоморфной функиии $g \in \mathscr{O}_{M, x}$, голоморфной $(q-1)$-формы $\xi \in \Omega_{M, x}^{q-1}$ и голоморфной q-формы $\eta \in \Omega_{M, x}^{q}$, такие, что

(i) $\operatorname{dim}_{\mathbb{C}} D \cap\{z \in M: g(z)=0\} \leqslant n-1$,

(ii) $g \omega=\frac{d h}{h} \wedge \xi+\eta$.

Обозначим через $\varrho: \Omega_{M, x}^{q}(\log D) \rightarrow \Omega_{M, x}^{q}$ гомоморфизм умножения на $h$, и пусть $\omega \in \Omega_{M, x}^{q}(\log D)$. Тогда существует элемент $g \in \mathscr{O}_{M, x}$ из леммы 2 , такой, что $g h \omega \in h \Omega_{M, x}^{q}+d h \wedge \Omega_{M, x}^{q-1}$, т. е. $g h \omega=0$ в $\Omega_{D, x}^{q}$. Поскольку росток $g$ определяет неделитель нуля в $\mathscr{O}_{D, x}$, это, в частности, означает, что $h \omega \in$ Tors $\Omega_{D, x}^{q}$, где через Tors $\Omega_{D, x}^{q}$ обозначается подмодуль кручения пучка регулярных $q$-дифференциалов. Таким образом, $\operatorname{Im}(\varrho) \subseteq \operatorname{Tors} \Omega_{D, x}^{q}$ (хотя на самом деле здесь имеется равенство). Если Tors $\Omega_{D, x}^{q}=0$, то росток $g$ из представления (ii) может быть только обратимым элементом, а потому

$$
\Omega_{M, x}^{q}(\log D) \cong \Omega_{M, x}^{q}+\frac{d h}{h} \wedge \Omega_{M, x}^{q-1} .
$$

На самом деле этот изоморфизм можно получить и без приведенных выше рассуждений, прямо воспользовавшись точной последовательностью для модулей кручения Tors $\Omega_{D, x}^{q}$ из работы [1] или [3].

Теорема 2. Пусть D-нормальная гиперповерхность. Тогда

$$
\operatorname{Ind}_{\text {hom }, D, x}(V)=\operatorname{dim} \mathscr{O}_{M, x} /\left(h, J_{x} \mathscr{V}\right)+\sum_{i=c^{\prime}}^{n+1}(-1)^{i} \operatorname{dim} H_{i}\left(\Omega_{D, x}^{\bullet}, \iota_{V}\right),
$$

где $c^{\prime}=2\left[\frac{c+1}{2}\right]+1$, квадратные скобки обозначают иелую часть, а сумма полагается равной нулю, если ниюний предел суммирования больше верхнего.

ДокАЗАтЕЛьство. Хорошо известно (см. [7]), что Tors $\Omega_{D}^{q}=0$ для $0<q<c$ (на самом деле это верно не только для гиперповерхностей, но и для полных пересечений). Вместе с изоморфизмом (9) это показывает, что $\Omega_{M, x}^{q} / h \Omega_{M, x}^{q}(\log D)$ $\cong \Omega_{D, x}^{q}$ для всех таких $q$. Поэтому из точной последовательности (2) следует, что

$$
H_{i}\left(\Omega_{D}^{q}, \iota_{V}\right) \cong H_{i-1}\left(\Omega_{D}^{q}[-1], \iota_{V}\right)=H_{i-2}\left(\Omega_{D}^{q}, \iota_{V}\right)
$$

для всех $i=3, \ldots, c+1$. В частности, в указанном диапазоне размерности групп $\iota_{V}$-гомологий комплекса $\Omega_{D, x}^{\bullet}$ из двух серий $H_{2 i}$ и $H_{2 i-1}$ одинаковы. Далее, нетрудно видеть, что точная последовательность $\left(7^{\circ}\right)$ получается из (7) с помощью точно таких же рассуждений, как в доказательстве теоремы 1, не только для свободных дивизоров Саито, но и для любых гиперповерхностей, обладающих свойством $H_{0}\left(\Omega_{M, x}^{\bullet} / h \Omega_{M, x}^{\bullet}(\log D), \iota_{\mathscr{V}}\right) \cong \mathscr{O}_{M, x} /\left(h, J_{x} \mathscr{V}\right)$. Для нормальных особенностей последнее выполнено ввиду формулы (9). Поэтому размерности групп $H_{1}$ и $H_{2}$ также равны, откуда и следует нужная формула. Целая часть при задании нижнего предела суммирования здесь понадобилась для того, чтобы различать случаи четной и нечетной коразмерности. 
СЛЕДСТВИЕ 4. Пусть $x \in D$ - изолированная особенность как гиперповерхности $D$, так и векторного поля $\mathscr{V} \in \operatorname{Der}(\log D), \mathscr{V}(h)=\varphi h u \varphi \in \mathscr{O}_{M, x}$. Тогда

$$
\operatorname{Ind}_{\text {hom }, D, x}(V)=\operatorname{dim} \mathscr{O}_{M, x} /\left(h, J_{x} \mathscr{V}\right)+\varepsilon \operatorname{dim} \operatorname{Ann}_{\mathscr{B}_{x}}(h) /(\varphi) \mathscr{B}_{x},
$$

где $\varepsilon=-1$, если $п$ четно, и $\varepsilon=0$ в противном случае.

ДокАЗАТЕЛЬСТВо. Поскольку для изолированной особенности $c=n$, из теоремы 2 получаем

$$
\operatorname{Ind}_{\text {hom }, D, x}(V)=\operatorname{dim} \mathscr{O}_{M, x} /\left(h, J_{x} \mathscr{V}\right)+\varepsilon \operatorname{dim} H_{n+1}\left(\Omega_{D, x}^{\bullet}, \iota_{V}\right) .
$$

Как показано в только что проведенном рассуждении с использованием точной последовательности $\left(7^{\circ}\right)$, группы четномерных и нечетномерных гомологий $H_{2 i}$ и $H_{2 i-1}$ комплекса $\Omega_{D, x}^{\bullet}$ вплоть до $H_{n+1}$ изоморфны группам $H_{2}$ и $H_{1}$ соответственно. Это завершает доказательство.

ЗАмЕчАниЕ 5. В работе [5] аналогичный результат для усеченного комплекса регулярных дифференциалов $\left(\Omega_{D}^{q}, \iota_{V}\right)_{q=0, \ldots, n}$ устанавливается прямым вычислением с использованием спектральных последовательностей, ассоциированных с конусом морфизма, а в работе [6] этот подход развивается для гиперповерхностей с одномерным подпространством особенностей, т. е. в случае $c=n-1$.

\section{§9. Индекс и регулярные мероморфные формы}

В заключение затронем еще один круг вопросов, тесно связанных с понятием вычета логарифмических форм. Обозначим $\mathscr{O}_{D}$-модуль ростков мероморфных функций на ростке гиперповерхности $D$ через $\mathscr{M}_{D}$. Тогда отображение вычета - это гомоморфизм $\mathscr{O}_{M}$-модулей

$$
\text { res: } \Omega_{M}^{q}(\log D) \rightarrow \mathscr{M}_{D} \otimes_{\mathscr{O}_{D}} \Omega_{D}^{q-1}
$$

который сопоставляет ростку $\omega \in \Omega_{M, x}^{q}(\log D)$ элемент $\frac{1}{g} \xi \in \mathscr{M}_{D, x} \otimes_{\mathscr{O}_{D, x}} \Omega_{D, x}^{q-1}$, заданный представлением (ii) из леммы 2. Этот элемент называется вычетом ростка $\omega$ и обозначается через $\operatorname{res}(\omega)$.

Пусть теперь $\omega_{D}^{n} \cong \operatorname{Ext}_{\mathscr{O}_{M}}^{1}\left(\mathscr{O}_{D}, \Omega_{M}^{n+1}\right)$ - дуализирующий модуль Гротендика для ростка $D \subset M$, который при наших предположениях локально изоморфен $\mathscr{O}_{D}$. Тогда элементы из $\operatorname{Hom}_{\mathscr{O}_{D}}\left(\Omega_{D}^{n-q}, \omega_{D}^{n}\right), q=0, \ldots, n$, называются регулярными мероморфными дифференциальными $q$-формами, а соответствующие пучки обозначаются через $\omega_{D}^{q}$ (см. $\left.[4,3]\right)$. Можно доказать (loc. cite), что $\omega_{D, x}^{0}$ содержит все ростки слабо голоморфных функций на $D$ (ср. $\left.[13,14]\right)$, или локально ограниченных мероморфных функций на $D$. Наконец, отметим, что стягивание $\iota_{V}$ на комплексе $\Omega_{D}^{q}$ по двойственности индуцирует канонический гомоморфизм $\mathscr{O}_{D}$-модулей $\iota_{V}: \omega_{D}^{q} \rightarrow \omega_{D}^{q-1}$ и тем самым наделяет $\omega_{D}^{\bullet}$ структурой комплекса.

ПРЕДЛОЖЕНИЕ 3. Пусть $D \subset M-$ росток произволъной гиперповерхности. Тогда существует точная последовательность комплексов $\mathscr{O}_{M}$-модулей

$$
0 \longrightarrow\left(\Omega_{M}^{\bullet}, \iota \mathscr{V}\right) \longrightarrow\left(\Omega_{M}^{\bullet}(\log D), \iota_{\mathscr{V}}\right) \stackrel{\text { res }}{\longrightarrow}\left(\omega_{D}^{\bullet}[-1], \iota_{V}\right) \longrightarrow 0,
$$

индуиирующая естественные изоморфизмы групп гомологий

$$
H_{q}\left(\Omega_{M, x}^{\bullet}(\log D), \iota \mathscr{V}\right) \cong H_{q}\left(\omega_{D, x}^{\bullet}[-1], \iota_{V}\right)=H_{q-1}\left(\dot{\omega}_{D, x}^{\bullet}, \iota_{V}\right)
$$


для всех $q \geqslant 2$.

ДокАЗАтЕЛьство. Из определений следует, что $\operatorname{res}\left(\iota_{\mathscr{V}}(\omega)\right)=-\iota_{\mathscr{V}}(\operatorname{res}(\omega))$, так что отображение вычета коммутирует с точностью до знака со стягиванием ı⿻ (равно как и с внешним дифференцированием $d$; см. [3]). Отсюда следует первое утверждение. Ацикличность комплекса $\left(\Omega_{M}^{\bullet}, \iota \mathscr{V}\right)$ в положительных размерностях дает искомые изоморфизмы и, кроме того, точную последовательность

$$
0 \rightarrow H_{1}\left(\Omega_{M}^{\bullet}(\log D), \iota \mathscr{V}\right) \rightarrow H_{0}\left(\omega_{D}^{\bullet}, \iota_{V}\right) \rightarrow H_{0}\left(\Omega_{M}^{\bullet}, \iota_{V}\right) \rightarrow H_{0}\left(\Omega_{M}^{\bullet}(\log D), \iota_{\mathscr{V}}\right) \rightarrow 0 .
$$

В частности, для свободных дивизоров Саито получаем описание $\iota_{V}$-гомологий комплекса регулярных мероморфных дифференциальных форм $\omega_{D}^{\bullet}$ в терминах комплекса Кошуля.

\section{ЛитерАТУрА}

1. Александров А. Г. О комплексе де Рама неизолированных особенностей. Функц. анализ и его прил., 22, вып. 2, 59-60 (1988).

2. Александров А. Г. Неизолированные особенности Саито. Матем. сб., 137 (179), № 4 (12), 554-567 (1988).

3. Aleksandrov A. G. Nonisolated hypersurface singularities. In: Adv. in Soviet Math. (Arnold V. I., ed.), Vol. 1, Amer. Math. Soc., Providence, RI, 1990, pp. 211-246.

4. Barlet $D$. Le faiseau $\omega_{X}^{\bullet}$ sur un espace analytique $X$ de dimension pure. Lecture Notes in Math., Vol. 670, 187-204, Springer-Verlag, 1978.

5. Gómez-Mont X. An algebraic formula for the index of a vector field on a hypersurface with an isolated singularity. J. Alg. Geom., 7, 731-752 (1998).

6. Giraldo L., Gómez-Mont X., Mardešić P. On the index of vector fields tangent to hypersurfaces with non-isolated singularities. J. London Math. Soc. (2), 65, No. 2, 418-438 (2002).

7. Greuel G.-M. Der Gauß-Manin-Zusammenhang isolierter Singularitäten von vollstandigen Durchschnitten. Math. Ann., 214, No. 3, 235-266 (1975).

8. Грифбитс Ф., Харрис Дж. Принципы алгебраической геометрии. Т. 2. Мир, М., 1982.

9. Хартсхорн Р. Алгебраическая геометрия. Мир, М., 1981.

10. Milnor J. Topology from the differential viewpoint. Princeton Univ. Press, Princeton, NJ, 1997.

11. Reiffen H.-J., Vetter U. Pfaffsche Formen auf komplexen Räumen. Math. Ann., 167, No. 4, 338-350 (1966).

12. Rossi H. Vector fields on analytic spaces. Ann. of Math. (2), 78, 455-467 (1963).

13. Saito $K$. On the uniformization of complements of discriminant loci. In: Hyperfunctions and Linear partial differential equations, RIMS Kōukyūroku, 287, 117-137 (1977).

14. Saito $K$. Theory of logarithmic differential forms and logarithmic vector fields. J. Fac. Sci. Univ. Tokyo, Sect. IA, Math., 27, No. 2, 265-291 (1980).

Институт проблем управления РАН email: alexandr@ipu.rssi.ru

Поступило в редакцию 3 марта 2004 г. 